\title{
Ressource oder Belastung?
}

\section{Die Bedeutung verschiedener Mentoringformen für das \\ Beanspruchungserleben angehender Lehrkräfte im Praxissemester}

\author{
Marike Bruns $\mathbb{D} \cdot$ Simon Küth $\cdot$ Daniel Scholl $\cdot$ Christoph Schüle
}

Eingegangen: 3. August 2020 / Überarbeitet: 17. April 2021 / Angenommen: 19. April 2021 / Online publiziert: 19. Mai 2021

(C) Der/die Autor(en) 2021

Zusammenfassung Für viele angehende Lehrkräfte stellt der Einstieg in den Lehrer/innenberuf ein normativ-kritisches Lebensereignis dar, das mit einer erhöhten Beanspruchung einhergehen kann. Um diesen Einstieg - unter anderem hinsichtlich des Beanspruchungserlebens - zu erleichtern, wurde in Deutschland flächendeckend das Praxissemester in das Lehramtsstudium eingeführt, in dem Studierende bei ihren Unterrichtsversuchen von erfahrenen Mentor/innen begleitet werden. Anders als beabsichtigt scheint das Mentoring im Praxissemester aber nicht ausschließlich beanspruchungsabpuffernde Effekte zu haben, sondern ebenfalls beanspruchungsinduzierend wirken zu können. Um diesen differentiellen Effekt des Mentorings aufzuklären, wird im Kontext der allgemeinen Belastungs- und Beanspruchungsmodelle für den Lehrer/innenberuf und aus der Perspektive der Selbstbestimmungstheorie angenommen, dass die Beziehungsform zwischen Mentees und Mentor/innen, wie sie sich unter anderem im transmissiven und konstruktivistischen Mentoring zeigt, einen Effekt auf die positiven und negativen Beanspruchungsreaktionen der Mentees hat, der über die wahrgenommene Basic-Need-Satisfaction vermittelt wird. Zur Untersuchung dieser Annahme wurden 177 Lehramtsstudierende im Praxissemester zu zwei Messzeitpunkten gebeten, sowohl ihre negativen Beanspruchungsreaktionen in Form ihrer emotionalen Erschöpfung als auch ihre positiven Beanspruchungsreaktionen in Form des beruflichen Enthusiasmus einzuschätzen. Außerdem wurde die Basic-Need-Satisfaction der angehenden Lehrkräfte zu beiden Messzeitpunkten sowie das transmissive und konstruktivistische Mentoring aus der Perspektive der Mentees zum zweiten Messzeitpunkt erfasst. Die Ergebnisse bestätigen den angenommenen Mediationseffekt teilweise. So vermittelt zwar die Veränderung der Basic-Need-Satisfaction den Zusammenhang zwischen beiden Mentoringformen und

M. Bruns $(\bowtie) \cdot$ S. Küth $\cdot$ D. Scholl $\cdot$ C. Schüle

Fakultät I, Arbeitsbereich Schulpädagogik und Allgemeine Didaktik, Universität Vechta, Driverstraße 22, 49377 Vechta, Deutschland

E-Mail: marike.bruns@uni-vechta.de 
der Veränderung des beruflichen Enthusiasmus vollständig, allerdings zeigt die Veränderung der Basic-Need-Satisfaction keinen Effekt auf die Veränderung der emotionalen Erschöpfung, die ihrerseits jedoch direkt durch das konstruktivistische und transmissive Mentoring vorhergesagt wird.

Schlüsselwörter Emotionale Erschöpfung · Mentoring · Praxissemester · BasicNeed-Satisfaction · Beruflicher Enthusiasmus

\title{
Resource or demand?
}

The impact of different mentoring approaches on student teachers' occupational well-being in a long-term student teaching internship

\begin{abstract}
For many prospective teachers the transition from university to school represents a major life event. As such, it may be accompanied by a reduced occupational well-being. In order to facilitate this transition for all prospective teachers, a long-term student teaching internship was introduced in most German higher teacher education programs. During this internship the student teachers are assigned to experienced mentors. However, despite good intentions, it seems as if the mentoring during the internship does not solely have positive effects on student teachers' occupational well-being but also negative ones. In order to explain these differential effects the study at hand assumes-based on general models of teachers' stress and strain and self-determination theory-that the effects of different mentoring approaches, e.g. transmissive and constructivist mentoring, on student teachers' occupational well-being are mediated by their basic need satisfaction. To test this assumption 177 student teachers were asked to assess their basic need satisfaction and their positive and negative occupational well-being in form of their emotional exhaustion and their work-related enthusiasm at two points in time. In addition, student teachers rated scales on transmissive and constructivist mentoring once during their internship. The results partially confirm the assumed mediation effects. Changes in student teachers' basic need satisfaction mediate the link between the two mentoring approaches and changes in their work-related enthusiasm, but changes in student teachers' basic need satisfaction do not affect changes in their emotional exhaustion. However, constructivist and transmissive mentoring directly predict changes in student teachers' emotional exhaustion.
\end{abstract}

Keywords Emotional exhaustion · Mentoring - Student teaching internship - Basic need satisfaction · Work enthusiasm

\section{Einleitung}

Der Berufseinstieg stellt für angehende Lehrkräfte ein normativ-kritisches Lebensereignis dar, das mit einer erhöhten Beanspruchung einhergehen kann (Dicke et al. 2015). Begünstigt werden kann eine solche Beanspruchungserhöhung, wenn das professionelle Wissen zum Zeitpunkt des Berufseinstiegs noch zu wenig handlungsund situationsbezogen organisiert ist, um die Schüler/innen in einem störungsar- 
men und wertschätzenden Unterrichtsklima kognitiv aktivieren zu können (Klusmann et al. 2012). Um angehenden Lehrkräften deshalb schon im Lehramtsstudium die Möglichkeit zu bieten, ihr theoretisches Wissen in realen Unterrichtssituationen praktisch anzuwenden und handlungsbezogen zu reorganisieren (König et al. 2020; Ulrich et al. 2020), wurde in Deutschland flächendeckend das Praxissemester in das Lehramtsstudium eingeführt (Rothland und Boecker 2015).

Dieses Praxissemester, das u.a. in der zeitlichen Verortung, der Dauer, der inhaltlichen Ausgestaltung und der Zielsetzung zwischen den Bundesländern variiert (Weyland 2012), ist ein Langzeitpraktikum, in dem Studierende über mehrere Wochen unterrichtspraktische Erfahrungen in Praktikumsschulen sammeln und in universitären Begleitveranstaltungen reflektieren. Im Unterschied zum Berufseinstieg, werden die angehenden Lehrkräfte im Praxissemester in ihren Lernprozessen sowohl von Dozierenden an den Universitäten als auch Mentor/innen, erfahrenen Lehrkräften an den Praktikumsschulen, unterstützt.

Bezogen auf die Wirkungen des Mentorings zeichnen die Forschungsbefunde allerdings ein ambivalentes Bild: Während die mentorielle Unterstützung durchaus positive Effekte auf die situations- und handlungsbezogene Qualität des Wissens der angehenden Lehrkräfte haben kann (König et al. 2020; Ulrich et al. 2020), kann sie aber auch zu einem Beanspruchungsanstieg während des Praxissemesters beitragen (Klassen und Durksen 2014; Krawiec et al. 2020). Ein solcher Effekt des Mentorings läuft jedoch einem der Ziele des Praxissemesters, der proaktiven Abpufferung des Beanspruchungserlebens beim Berufseinstieg, zuwider.

Ein möglicher Erklärungsansatz für diesen Effekt könnte in der Beziehungsqualität zwischen angehenden Lehrkräften und ihren Mentor/innen liegen (Ellis et al. 2020). Denn diese Qualität könnte die wahrgenommene Befriedigung der menschlichen Grundbedürfnisse nach Autonomie, Kompetenz und Zugehörigkeit (BasicNeed-Satisfaction) beeinflussen (Hoffmann und Springer 2014; Simões und Alarcão 2014) und darüber vermittelt einen Effekt auf die Beanspruchung von Praxissemesterstudierenden haben. Um diesen Mediationseffekt zu prüfen, werden in der vorliegenden Studie die Formen des konstruktivistischen und transmissiven Mentorings (Richter et al. 2013) untersucht. Dabei wird auf der Grundlage von Rahmenmodellen zur Belastung und Beanspruchung im Lehrer/innenberuf (Böhm-Kasper 2004; Landwehr et al. 1983; Rudow 1994) und aus der Perspektive der Selbstbestimmungstheorie (Ryan und Deci 2017) gefragt, ob die jeweils implizierte Beziehungsqualität dieser beiden Formen indirekt - vermittelt durch die Basic-Need-Satisfaction - einen Effekt auf die positiven und negativen Beanspruchungsreaktionen von Praxissemesterstudierenden hat.

\section{Theoretischer Hintergrund}

Unter Rückgriff auf den Erklärungsansatz zur schulischen Belastung von Landwehr et al. (1983), das Rahmenmodell zur schulischen Belastung und Beanspruchung von Böhm-Kasper (2004) und das Rahmenkonzept zur Belastungs-BeanspruchungsSequenz in der Lehrtätigkeit (Rudow 1994) lassen sich objektive und subjektive Belastungen voneinander abgrenzen. Als objektive Belastungen gelten die Arbeits- 
aufgaben von Lehrkräften und die Bedingungen, unter denen sie erfüllt werden. Als subjektive Belastungen werden dagegen die Belastungen definiert, die aus der Deutung der objektiven Belastungen hervorgehen und tatsächlich von einer Person erlebt werden. Wenn die Belastungen die eigenen Bewältigungsmöglichkeiten übersteigen, können aus subjektiven Belastungen kurzfristige Beanspruchungsreaktionen und langfristige Beanspruchungsfolgen resultieren, die - je nach Bewältigungsmöglichkeiten einer Person - positiv oder negativ ausfallen können (Rudow 1994). Übersteigen die Belastungen die Bewältigungsmöglichkeiten nur moderat, werden sie als Herausforderung mit entsprechenden positiven Beanspruchungsreaktionen wie Enthusiasmus (Keller et al. 2014) und langfristigen Folgen wie Zufriedenheit oder einem gestärkten Gefühl der eigenen Handlungskompetenz erlebt. Führen die vorhandenen Bewältigungsmöglichkeiten allerdings zu einer deutlichen Über- oder Unterforderung, treten eher negative emotionale Beanspruchungsreaktionen wie Angst, Stress oder Erschöpfung auf, die langfristig in chronische Beanspruchungsfolgen wie das Burnout-Syndrom münden können (Rudow 1994).

Erste Studien zum Praxissemester zeigen, dass angehende Lehrkräfte dort auf ein hohes Maß an objektiven Belastungen treffen (Krawiec et al. 2020). In Abhängigkeit von den vorhandenen Bewältigungsmöglichkeiten der Studierenden können diese Belastungen zu positiven oder negativen Beanspruchungsreaktionen und -folgen führen (Darge et al. 2018; Kücholl et al. 2019; Römer et al. 2018), weshalb das Praxissemester insgesamt als eine sensible Beanspruchungsphase einzuschätzen ist (Schüle et al. 2017b).

Ein Teil dieser Beanspruchungsreaktionen und -folgen bezieht sich dabei u a. auf das Mentoring als Aspekt der Sozialbeziehungen, die einen objektiven Belastungsfaktor darstellen (Böhm-Kasper 2004; Landwehr et al. 1983). Das Mentoring kann einen beanspruchungsreduzierenden Effekt haben (Kücholl et al. 2019; Römer et al. 2018), es kann sich aber auch negativ auf die Beanspruchungsreaktionen angehender Lehrkräfte auswirken (Klassen und Durksen 2014; Krawiec et al. 2020), z.B. weil die Freude an der Schulpraxis im Verlauf des Praxissemesters abnehmen kann, wenn die soziale Unterstützung durch die Mentor/innen von den Studierenden als Hindernis gedeutet wird (Darge et al. 2018).

Da die Qualität des Mentorings über eine eher anerkennende und eine eher hierarchische Form z.B. in den Varianten des konstruktivistischen und transmissiven Mentorings (Richter et al. 2013) zu variieren scheint (Ellis et al. 2020), lässt sich annehmen, dass ein möglicher Grund für die Differentialität dieser Auswirkungen in der Qualität der Mentor/innen-Mentee-Beziehung liegt: Eine anerkennende Beziehungsform, die insbesondere dem konstruktivistischen Mentoring zugrunde liegt, basiert auf den Zielen, Wünschen und Idealen der angehenden Lehrkräfte (Richter et al. 2013) und wird in einer respektvollen, entgegenkommenden und reflektierenden Atmosphäre (Hennissen et al. 2008) durch individuelle Begleitung mit einer nicht-kontrollierenden und informativen Sprache verwirklicht (Reeve 2009). In einer hierarchischen Beziehungsform, die sich eher im transmissiven Mentoring zeigt, geben Mentor/innen den Mentees dagegen ihre eigenen Ziele, Wünsche und Ideale vor (Richter et al. 2013) und lenken die Mentees durch eine kontrollierende und direktive Sprache (Reeve 2009) in die Richtung dieser Vorgaben, sodass Mentor/ 
innen den Studierenden nur sehr wenig oder keinen Raum für die Erkundung und Verwirklichung eigener Ziele, Wünsche und Ideale lassen (Ellis et al. 2020).

Die Deutung dieser beiden Beziehungsformen durch die Studierenden - so lässt sich auf der Grundlage des transaktionalen Modells von Lazarus (1999) ferner annehmen - umfasst zwei Aspekte: einerseits die Bewertung hinsichtlich der Frage, inwieweit das Mentoring als objektive Belastung das Erreichen individuell bedeutsamer Ziele fördert oder behindert (primäre Bewertung) und andererseits die Beurteilung der individuellen Bewältigungsmöglichkeiten, die den Studierenden für die Zielverfolgung unter den gegebenen objektiven Belastungen zur Verfügung stehen (sekundäre Bewertung). Diese individuell bedeutsamen Ziele können wiederum auf der Basis der Selbstbestimmungstheorie im menschlichen Streben nach der Befriedigung der angeborenen Grundbedürfnisse nach Autonomie, Kompetenz und Zugehörigkeit gesehen werden (Ryan und Deci 2017). Da die Befriedigung dieser Grundbedürfnisse einen Einfluss auf die Bewertung der zur Verfügung stehenden Bewältigungsmöglichkeiten haben kann (Haslam et al. 2018), könnte der Grad der Bedürfnisbefriedigung den Einfluss einer objektiven Belastung auf die subjektive Belastung und ihren positiven und negativen Beanspruchungsreaktionen und -folgen vermitteln (Aldrup et al. 2017; Fernet et al. 2013; Van den Broeck et al. 2008).

Unter diesen Prämissen lässt sich für die Wirkung der anerkennenden Beziehungsform vermuten, dass die Möglichkeit der Verwirklichung eigener Ziele, Wünsche und Ideale in einer respektvollen, entgegenkommenden und reflektierenden Atmosphäre die Basic-Need-Satisfaction der Mentees stärkt, wodurch die Mentees mehr positive und weniger negative Beanspruchungsreaktionen erleben sollten. Dagegen sollte die begrenzte Möglichkeit der Verwirklichung eigener Ziele, Wünsche und Ideale sowie die kontrollierende und direktive Sprache des hierarchischen Mentorings die Basic-Need-Satisfaction der Mentees einschränken und zu mehr negativen und weniger positiven Beanspruchungsreaktionen führen.

Obschon erste Studien zeigen, dass das erlebte Mentoring in den Praxisphasen des Studiums einen Einfluss auf die Basic-Need-Satisfaction der Mentees haben (Hoffmann und Springer 2014; Simões und Alarcão 2014) und dass diese BasicNeed-Satisfaction - unabhängig vom Mentoring - einen Einfluss auf die positiven und negativen Beanspruchungsreaktionen und -folgen haben kann (Aldrup et al. 2017; Fernet et al. 2013; Van den Broeck et al. 2008), wurde der beschriebene indirekte Effekt bislang nicht empirisch geprüft. Deshalb wird in der vorliegenden Studie untersucht, inwiefern die Effekte der anerkennenden Beziehungsform im konstruktivistischen Mentoring und der hierarchischen Beziehungsform im transmissiven Mentoring auf die positiven und negativen Beanspruchungsreaktionen von Praxissemesterstudierenden durch deren Basic-Need-Satisfaction vermittelt werden.

\section{Methode}

\subsection{Untersuchungsdesign}

Die Grundlage der vorliegenden Studie bildet die Evaluation des Praxissemesters an der Universität Vechta in Niedersachsen, das im Masterstudium für das Lehr- 
amt verortet ist und 18 Wochen dauert (Niedersächsisches Kultusministerium 2015; $\S 9)$. In diesem Semester verbringen die Studierenden vier Schultage pro Woche (insgesamt mindestens 15 Zeitstunden) an einer zugewiesenen Praktikumsschule und jeweils einen Tag in der Universität. Die Studierenden werden von einem Lehrtandem betreut, das aus einem/r Fachdidaktiker/in der Universität (erste Phase der Lehramtsausbildung) und einer Seminarleitung aus einem Studienseminar (zentrale Ausbildungsinstitution des Vorbereitungsdienstes in der zweiten Phase der Lehramtsausbildung) besteht. Dieses Lehrtandem besucht jeweils zwei eigenständig vorbereitete Unterrichtsstunden der Studierenden pro studiertem Unterrichtsfach. In der Schule werden die Studierenden während des gesamten Praxissemesters von mindestens einem/r Mentor/in bei ihrer fachspezifischen Planung, Durchführung und Analyse der Unterrichtsversuche begleitet. Diese/r Mentor/in, die/der regelmäßiges Feedback zur individuellen Kompetenzentwicklung gibt, wird von der Schulleitung der Praktikumsschule bestimmt. Um auf diese Begleitungsaufgaben vorbereitet zu werden, nehmen die Mentor/innen an einer eintägigen Fortbildungsveranstaltung teil, die eine allgemeine Einführung zur Gestaltung von Beratungs- und Reflexionsgesprächen und effektiven Unterrichtshospitationen, Workshops zu Merkmalen eines lernwirksamen Unterrichts und Angebote zu Themen wie dem Forschenden Lernen oder der Gesprächsführung in schwierigen Situationen umfasst.

In den ersten beiden Praktikumswochen hospitieren die Studierenden pro Fach zunächst in ca. 45 Unterrichtstunden ihrer Mentor/innen. Ab der dritten Praktikumswoche planen und gestalten die Studierenden zunehmend eigene Unterrichtsversuche (insgesamt $32 \mathrm{~h}$ pro Fach), wovon pro Unterrichtsfach mindestens $16 \mathrm{~h}$, die in der Verantwortung des/r Mentor/innen stehen, in schriftlichen Unterrichtsentwürfen dokumentiert werden.

Die Studierenden wurden an drei Messzeitpunkten zu ihrem motivational-affektiven Erleben und ihrer selbstregulativen Kompetenz befragt (Scholl et al. 2020). In Bezug auf ihr motivational-affektives Erleben wurden die Studierenden zu allen Messzeitpunkten gebeten, ihre emotionale Erschöpfung, ihren beruflichen Enthusiasmus und ihre individuelle Basic-Need-Satisfaction einzuschätzen. Zum zweiten und dritten Messzeitpunkt beurteilten sie außerdem die erlebte Beziehungsqualität des Mentorings. Der erste Messzeitpunkt $\left(t_{1}\right)$ lag in der Auftaktveranstaltung zum Praxissemester. Für die zweite Messung $\left(\mathrm{t}_{2}\right)$ wurde der „Pädagogische Tag“ genutzt, den die Universität Vechta für alle Praxissemesterstudierenden neun Wochen nach Beginn ihres Praxissemesters ausrichtet. Ausgehend von zwei Impulsvorträgen setzen sich die Studierenden an diesem Tag in verschiedenen Workshops mit fachspezifischen und fächerübergreifenden Möglichkeiten erfolgreichen Lehrens und Lernens auseinander. Der dritte Messzeitpunkt $\left(t_{3}\right)$ lag in den Nachbereitungsseminaren, die die Fachdidaktiken im Anschluss an die 18-wöchige Zeit in der Schule durchführen.

Um eine Konfundierung des Effektes der mentoriellen Lernbegleitung mit dem Lehrtandem zu vermeiden, das die Unterrichtsversuche ab dem zweiten Messzeitpunkt ebenfalls hospitiert und den Studierenden eine Rückmeldung zu ihrer Kompetenzentwicklung gibt, wurden zur Beantwortung der Forschungsfrage ausschließlich die Messzeitpunkte $t_{1}$ und $t_{2}$ betrachtet. 


\subsection{Untersuchungsinstrumente}

Zur Messung der negativen Beanspruchungsreaktion der Studierenden wurde die emotionale Erschöpfungsskala des Maslach Burnout Inventorys (Maslach und Jackson 1996) genutzt. Die Erfassung der positiven Beanspruchungsreaktion erfolgte mit der beruflichen Enthusiasmusskala von Keller et al. (2014). Für die Erhebung der Basic-Need-Satisfaction wurden die Studierenden gebeten, ihr unterrichtsbezogenes Autonomie-, Kompetenz- und Zugehörigkeitserleben auf einer Skala von Evelein et al. (2008) zu beurteilen. Die Operationalisierung des anerkennenden und hierarchischen Mentorings basierte auf den konstruktivistischen und transmissiven Mentoringskalen von Richter et al. (2013) (vgl. Tab. 1).

Tab. 1 Übersicht über die (Sub-)skalen zur Messung der einzelnen Konstrukte

\begin{tabular}{lllllll}
\hline & $N$ & Itembeispiel & Antwortskala & $\omega_{\mathrm{t} 1}$ & $\omega_{\mathrm{t} 2}$ & Quelle \\
\hline Emotionale & 9 & „Ich fühle mich von meiner Arbeit & $(1)$ trifft nicht zu & 0,87 & 0,83 & (Maslach \\
Erschöp- & & in der Schule ausgelaugt.“ & $\ldots$ & & & und \\
fung & & & $(5)$ trifft zu & & & Jackson \\
& & & & & 1996)
\end{tabular}

\section{Beruflicher Enthusiasmus}

\begin{tabular}{|c|c|c|c|c|c|c|}
\hline $\begin{array}{l}\text { Positiver } \\
\text { Affekt }\end{array}$ & 3 & $\begin{array}{l}\text { „Mir macht das Unterrichten mei- } \\
\text { nes Faches großen Spaß.“ }\end{array}$ & $\begin{array}{l}\text { (1) stimme gar } \\
\text { nicht zu }\end{array}$ & 0,80 & 0,84 & $\begin{array}{l}\text { (Keller } \\
\text { et al. }\end{array}$ \\
\hline $\begin{array}{l}\text { Emotionale } \\
\text { Expressi- } \\
\text { vität }\end{array}$ & 8 & $\begin{array}{l}\text { „Während des Unterrichts lache } \\
\text { ich oft.“ }\end{array}$ & $\begin{array}{l}\text { (5) stimme } \\
\text { vollständig zu }\end{array}$ & 0,75 & 0,68 & 2014) \\
\hline \multicolumn{7}{|c|}{ Basic-Need-Satisfaction } \\
\hline $\begin{array}{l}\text { Autonomie- } \\
\text { erleben }\end{array}$ & 4 & $\begin{array}{l}\text { „Wenn ich an meine Unterrichts- } \\
\text { stunden zurückdenke, dann habe } \\
\text { ich das Gefühl, dass meine Ent- } \\
\text { scheidungen mein ,wahres Ich“ } \\
\text { ausdrückten.“ }\end{array}$ & $\begin{array}{l}\text { (1) stimmt gar } \\
\text { nicht } \\
\ldots \\
\text { (5) stimmt } \\
\text { vollständig }\end{array}$ & 0,79 & 0,84 & $\begin{array}{l}\text { (Evelein } \\
\text { et al. } \\
2008)\end{array}$ \\
\hline $\begin{array}{l}\text { Kompetenz- } \\
\text { erleben }\end{array}$ & 5 & $\begin{array}{l}\text { „Wenn ich an meine Unterrichts- } \\
\text { stunden zurückdenke, dann habe } \\
\text { ich das Gefühl, dass ich mich gut } \\
\text { im Unterrichten ausgebildet fühl- } \\
\text { te.“ }\end{array}$ & & 0,85 & 0,82 & \\
\hline $\begin{array}{l}\text { Zugehörig- } \\
\text { keitserleben }\end{array}$ & 4 & $\begin{array}{l}\text { „Wenn ich an meine Unterrichts- } \\
\text { stunden zurückdenke, dann ha- } \\
\text { be ich das Gefühl, dass ich eine } \\
\text { Bindung zu den Schüler/innen } \\
\text { aufbauen konnte.“" }\end{array}$ & & 0,92 & 0,93 & \\
\hline \multicolumn{7}{|l|}{ Mentoringform } \\
\hline $\begin{array}{l}\text { Konstruktivis- } \\
\text { tisches } \\
\text { Mentoring }\end{array}$ & & $\begin{array}{l}\text { „Mein/e Mentor/innen unterstützen } \\
\text { mich darin, verschiedene Unter- } \\
\text { richtsmethoden auszuprobieren.“ }\end{array}$ & $\begin{array}{l}\text { (1) trifft nicht zu } \\
\ldots \\
\text { (6) trifft zu }\end{array}$ & - & 0,91 & $\begin{array}{l}\text { (Richter } \\
\text { et al. } \\
\text { 2013) }\end{array}$ \\
\hline $\begin{array}{l}\text { Transmissives } \\
\text { Mentorings }\end{array}$ & 5 & $\begin{array}{l}\text { „Mein/e Mentor/innen geben mir } \\
\text { vor, was ich verbessern muss.“ }\end{array}$ & & - & 0,83 & \\
\hline
\end{tabular}

$N$ Anzahl der Items, $\omega$ McDonalds Omega, $t_{l}$ erster Messzeitpunkt, $\mathrm{t}_{2}$ zweiter Messzeitpunkt 


\subsection{Stichprobe}

Aus der Grundgesamtheit von 253 zum Praxissemester angemeldeten Lehramtsstudierenden gaben zum ersten Messzeitpunkt $n=245$ Studierende ihr Einverständnis zur Teilnahme an der Studie, von denen $n=177(72,2 \%)$ am zweiten und $n=183$ $(74,7 \%)$ am dritten Messzeitpunkt teilnahmen. Der Rücklauf $(73,5 \%)$ liegt damit oberhalb des Rücklaufs vergleichbarer Längsschnittstudien mit mehr als zwei Messzeitpunkten (siehe für einen Überblick: Schüle et al. 2017a).

Zum ersten Messzeitpunkt waren 79,4\% der Studierenden weiblich und 20,6\% männlich. Ihr durchschnittliches Alter betrug $M=25,27$ (SD=6,47) Jahre. Die Mehrzahl der Teilnehmer/innen befand sich im achten Fachsemester $(M=8 ; S D=1,44)$ und strebte eine spätere Tätigkeit an einer Grund- (77,1\%), Real- $(20 \%)$ oder Oberschule $(16,3 \%)$ an. Entsprechend ihrer Schulformwünsche absolvierte die Mehrheit der Studierenden ihr Praxissemester an einer Grundschule (75,5\%), gefolgt von Ober- $(14,7 \%)$, Real- $(6,1 \%)$ und Hauptschule $(2 \%)$.

Zur Untersuchung des Drop-Outs von $\mathrm{t}_{1} \mathrm{zu} \mathrm{t}_{2}$ wurde eine logistische Regressionsanalyse durchgeführt, die die Teilnahme an $t_{2}$ aus den demografischen Variablen Geschlecht, Alter, Fachsemester und Schulformwunsch sowie den interessierenden Konstrukten zu t $\mathrm{t}_{1}$ (Basic-Need-Satisfaction, emotionale Erschöpfung und beruflicher Enthusiasmus) vorhersagt $\left(\chi^{2}(7)=16,49 ; p<0,05\right.$; Nagelkerkes $\left.\mathrm{R}^{2}=0,10\right)$. Einzig die Variable Geschlecht prädizierte die Teilnahme an $\mathrm{t}_{2}$ signifikant (Wald $=6,37$; $\mathrm{df}=1$; $\left.p<0,05 ; \operatorname{Exp}(\mathrm{B})=2,61 ; \mathrm{CI}_{95} \%:[1,24 ; 5,48]\right)$. Allerdings konnte weder für die emotionale Erschöpfung $(\mathrm{t}(241)=-1,95, p=\mathrm{n}$. s.) noch den beruflichen Enthusiasmus $(\mathrm{t}(241)=1,62, p=\mathrm{n} . \mathrm{s}$. $)$ oder die Basic-Need-Satisfaction $(\mathrm{t}(241)=-1,67, p=\mathrm{n} . \mathrm{s}$. ein signifikanter Unterschied zwischen den Geschlechtern $\mathrm{zu} \mathrm{t}_{1}$ festgestellt werden. Um die Verzerrung dennoch zu beachten, wurde das Geschlecht als Kovariate in allen Modellspezifikationen aufgenommen, und fehlende Werte wurden mittels des Full-Information-Maximum-Likelihood-Verfahrens berücksichtigt.

\subsection{Statistische Analysen}

Alle statistischen Analysen erfolgten mithilfe der Software Mplus 7.3 (Muthén und Muthén 2014). Die zeitliche Veränderung der einzelnen Konstrukte wurde zunächst mit drei Latent-Change-Modellen analysiert. Auf deren Basis wurde für die einzelnen Konstrukte jeweils ein messfehlerbereinigter Differenzwert berechnet, der auf seine Signifikanz und interindividuelle Variabilität hin untersucht wurde (McArdle 2009). Um die interindividuelle Variabilität der zeitlichen Veränderung auf der Ebene der einzelnen Studierenden näher zu beschreiben, wurde zusätzlich für jede Person ein individueller Differenzwert auf Basis der plausible values berechnet und dessen Signifikanz auf Individualebene geprüft (Asparouhov und Muthén 2010). Dieses Vorgehen ermöglicht jeweils die Angabe des Anteils an Studierenden, die einen Rückgang, Anstieg oder keine Veränderung in den einzelnen Konstrukten aufweisen.

Um anschließend einerseits untersuchen zu können, inwiefern das konstruktivistische und transmissive Mentoring interindividuelle Unterschiede in den intraindividuellen Veränderungen der emotionalen Erschöpfung, des beruflichen Enthusiasmus 
und der Basic-Need-Satisfaction erklärt und um andererseits die angenommenen Mediatoreffekte zu prüfen, wurden die einzelnen Latent-Change-Modelle in ein querschnittlich angelegtes Parallel-Process-Modell überführt (MacKinnon et al. 2007). Auf dessen Basis wurden für die Analyse der Art der Mediationseffekte vier ineinander geschachtelte Modelle spezifiziert und mittels des $\chi^{2}$-Differenzentests verglichen. In Modell 1 (direktes Effektmodell) wurden nur die direkten Effekte des konstruktivistischen und transmissiven Mentorings auf die latenten Differenzvariablen der Basic-Need-Satisfaction, der emotionalen Erschöpfung und des beruflichen Enthusiasmus betrachtet. In Modell 2 (partielles Mediationsmodell) wurden zusätzlich die Pfade der latenten Differenzvariablen der Basic-Need-Satisfaction auf die latenten Differenzvariablen der emotionalen Erschöpfung und des beruflichen Enthusiasmus spezifiziert. Auf Basis von Modell 2 wurden anschließend zwei vollständige Mediationsmodelle - jeweils getrennt für die emotionale Erschöpfung und den beruflichen Enthusiasmus - berechnet. In Modell 3 (vollständiges Mediationsmodell für die emotionale Erschöpfung) wurden ausschließlich die Pfade des konstruktivistischen und transmissiven Mentorings auf die Differenzvariable der emotionalen Erschöpfung auf Null restringiert, wohingegen die Pfade der beiden Mentoringformen auf die Differenzvariable des beruflichen Enthusiasmus frei geschätzt wurden. In Modell 4 (vollständiges Mediationsmodell für den beruflichen Enthusiasmus) wurden dagegen die Pfade der beiden Mentoringformen auf die Differenzvariable des beruflichen Enthusiasmus auf Null restringiert und deren Effekte auf die Differenzvariable der emotionalen Erschöpfung frei geschätzt. Um zuverlässige Signifikanzwerte für die einzelnen indirekten Effekte zu erhalten, wurden die Mediationsmodelle mithilfe des Bootstrapping-Verfahrens und 5000 Bootstrap-Samples geschätzt sowie die 95\%igen Konfidenzintervalle der indirekten Effekte betrachtet (MacKinnon et al. 2007). In allen Modellen wurde die Geschlechtszugehörigkeit als Kovariate berücksichtigt.

Aufgrund der hohen Interkorrelation der einzelnen Subskalen wurden diese als manifeste Indikatoren für die latente Modellierung des beruflichen Enthusiasmus und der Basic-Need-Satisfaction genutzt (vgl. Abb. 1). Die latenten Modellierungen der emotionalen Erschöpfung sowie des transmissiven und konstruktivistischen Mentorings erfolgten dagegen mit Item-Parcels. Die Item-Parcels wurden unter Verwendung des Item-to-construct-balance-Ansatzes gebildet, indem die Items mit hohen und niedrigen Faktorladungen gleichmäßig auf die Parcels verteilt wurden (Little et al. 2013) (vgl. Abb. 1). In den Messinvarianzanalysen zeigten sich die gewählten Indikatorisierungen der latenten Konstrukte als hinreichend vergleichbar über die Zeit, die konfirmatorischen Faktorenanalysen weisen zum Teil einen schlechten Modellfit auf.

Gemäß den Empfehlungen von Hu und Bentler (1999) wurden für die Evaluation der Modellgüte neben der $\chi^{2}$-Teststatistik auch der Comparative Fit-Index (CFI) und der Root Mean Square Error of Approximation (RMSEA) betrachtet. Allen Analysen lag das $5 \%$ ige Signifikanzniveau zugrunde. 

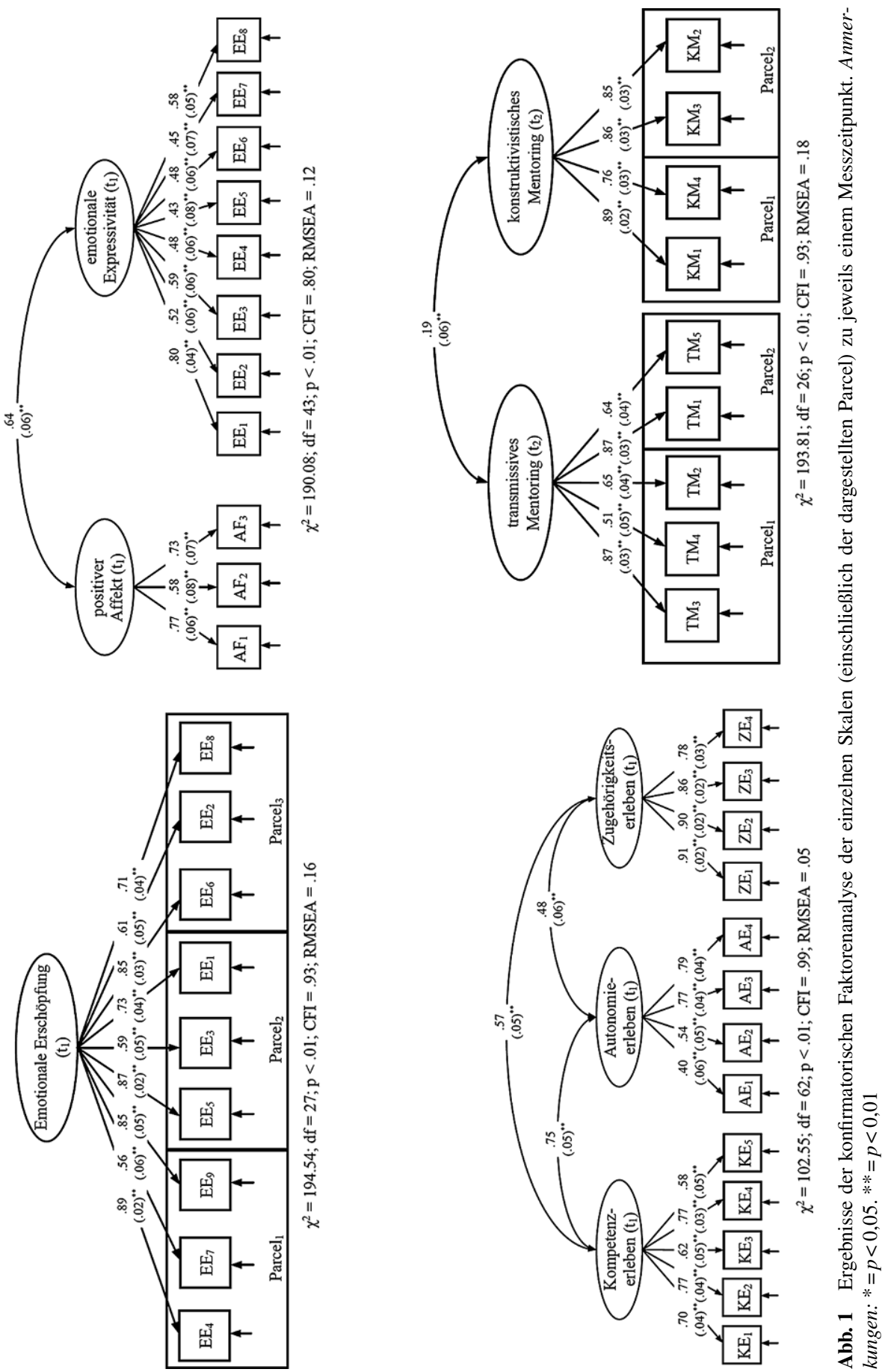


\section{Ergebnisse}

Tab. 2 enthält die Ergebnisse der einzelnen Latent-Change-Analysen einschließlich der - auf den individuellen Differenzwerten der plausible values basierenden - Angaben des jeweiligen Anteils an Studierenden, die einen Rückgang, Anstieg oder keine Veränderung in den Konstrukten aufweisen. Bei einer guten Modellqualität deutet sich für das latente Konstrukt der Basic-Need-Satisfaction ein signifikanter Zuwachs über die Zeit an. Dagegen erweisen sich die latenten Konstrukte der emotionalen Erschöpfung und des beruflichen Enthusiasmus bei einer guten Modellgüte als zeitstabil. In den latenten Varianzschätzungen zeigen sich daneben über alle Konstrukte hinweg bedeutsame interindividuelle Unterschiede zwischen den Studierenden sowohl $\mathrm{zu} \mathrm{t}_{1}$ als auch in der Veränderung $\mathrm{zu} \mathrm{t}_{2}$. In Bezug auf diese Unterschiede zeigen $6,2 \%$ der Studierenden in der intraindividuellen Differenzvariablen der emotionalen Erschöpfung einen bedeutsamen Anstieg und 82,7\% bzw. 11,1\% keine oder eine rückläufige Veränderung. Bei 7,4\% der Studierenden deutet sich ein signifikanter Zuwachs in ihrem beruflichen Enthusiasmus an, wohingegen bei 91,4\% keine und bei $1,2 \%$ eine negative Veränderung des beruflichen Enthusiasmus zu verzeichnen ist. Bei 44,4\% der Studierenden lässt sich eine deutliche Zunahme ihrer Basic-NeedSatisfaction beobachten. Diesem Zuwachs stehen insgesamt 54,7\% bzw. 0,8\% der Studierenden mit keiner Veränderung oder einer Abnahme ihrer Basic-Need-Satisfaction gegenüber.

Die Vergleiche des direkten Effektmodells (Modell 1: $\chi^{2}=341,33$; df =169; $p<0,01$; $\mathrm{CFI}=0,90$; RMSEA $=0,07)$ und der beiden vollständigen Mediationsmodelle (Modell 3: $\chi^{2}=316,26 ; \mathrm{df}=169 ; p<0,01 ; \mathrm{CFI}=0,91 ; \mathrm{RMSEA}=0,06$; Modell 4: $\chi^{2}=291,62 ; \mathrm{df}=169 ; p<0,01$; CFI=0,93; RMSEA=0,06) mit dem partiellen Mediationsmodell (Modell 2: $\chi^{2}=291,34 ; \mathrm{df}=167 ; p<0,01 ; \mathrm{CFI}=0,93$; RMSEA $=0,06)$ zeigen eine signifikant bessere Passung des vollständigen Mediationsmodells für den beruflichen Enthusiasmus (Modell 1 vs. Modell 2: $\Delta \chi^{2}(2)=$ 49,99**; Modell 2 vs. Modell 3: $\Delta \chi^{2}(2)=24,92^{* *}$; Modell 2 vs. Modell 4: $\Delta \chi^{2}(2)=$ $\left.0,28^{\text {n.s. }}\right)$.

Das vollständige Mediationsmodell für den beruflichen Enthusiasmus ist in Abb. 2 dargestellt. Im Rahmen dieses Modells kann das konstruktivistische Mentoring zu $\mathrm{t}_{2}$ bedeutsame interindividuelle Unterschiede sowohl in der intraindividuellen Veränderung der Basic-Need-Satisfaction $(\beta=0,24 ; S E=0,10 ; p<0,05)$ als auch in der intraindividuellen Veränderung der emotionalen Erschöpfung $(\beta=-0,32 ; S E=0,10$; $p<0,01)$ erklären. Das transmissive Mentoring $\mathrm{zu} \mathrm{t}_{2}$ sagt dagegen interindividuelle Unterschiede in der intraindividuellen Veränderung der Basic-Need-Satisfaction $(\beta=-0,25 ; S E=0,11 ; p<0,05)$ bedeutsam negativ und interindividuelle Unterschiede in der intraindividuellen Veränderung der emotionalen Erschöpfung $(\beta=0,39$; $S E=0,12 ; p<0,01)$ bedeutsam positiv vorher. Darüber hinaus klären die interindividuellen Unterschiede in der intraindividuellen Veränderung der Basic-Need-Satisfaction signifikant interindividuelle Unterschiede in der intraindividuellen Veränderung des beruflichen Enthusiasmus $(\beta=0,77 ; S E=0,16 ; p<0,01)$ auf. Die interindividuellen Unterschiede in der intraindividuellen Veränderung der Basic-Need-Satisfaction prädizieren jedoch keine interindividuellen Unterschiede in der intraindividuellen Veränderung der emotionalen Erschöpfung ( $\beta=0,05 ; S E=0,13 ; p=$ n. s.). 


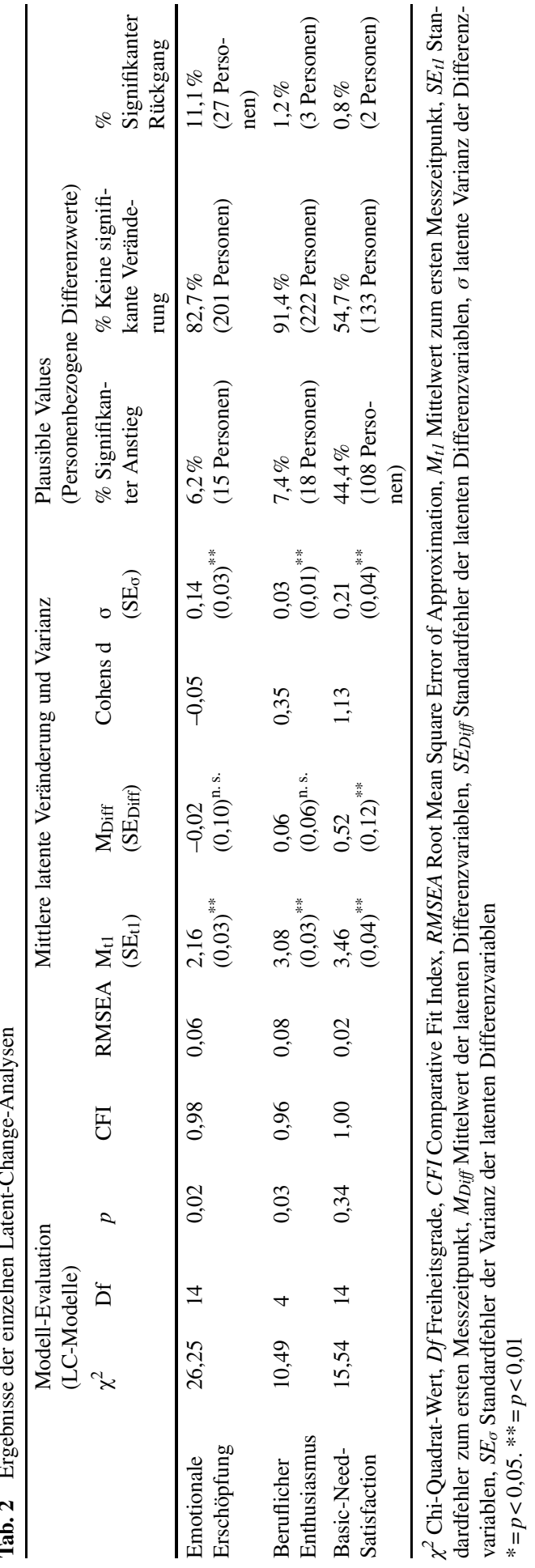




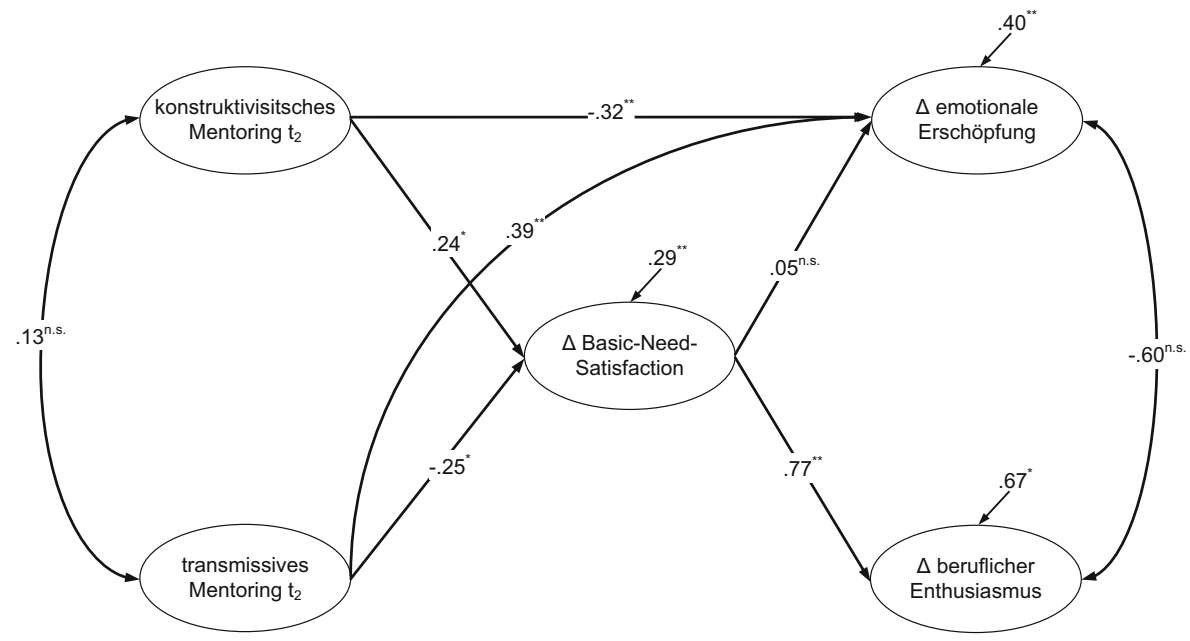

Abb. 2 Ergebnisse des vollständigen Mediationsmodells für den beruflichen Enthusiasmus. Anmerkungen: $\Delta$ latente Differenz, $n$. s. nicht signifikant. ${ }^{*}=p<0,05 ;{ }^{* *}=p<0,01$

Die Mediatoranalysen zeigen für das konstruktivistische Mentoring einen bedeutsamen indirekten Effekt auf die interindividuellen Unterschiede in der intraindividuellen Veränderung des beruflichen Enthusiasmus, der vollständig durch die interindividuellen Unterschiede in der intraindividuellen Veränderung der Basic-NeedSatisfaction mediiert wird ( $\beta_{\text {ind }}=0,19 ; S E_{\text {ind }}=0,08 ; p<0,05 ; \mathrm{CI}_{95} \%$ : $[0,06 ; 0,39]$ ). Für das transmissive Mentoring ist auch der durch die interindividuellen Unterschiede in der intraindividuellen Veränderung der Basic-Need-Satisfaction vollständig vermittelte indirekte Effekt auf die interindividuellen Unterschiede in der intraindividuellen Veränderung des beruflichen Enthusiasmus statistisch bedeutsam $\left(\beta_{\text {ind }}=-0,20\right.$; $\left.S E_{\text {ind }}=0,09 ; p<0,05 ; \mathrm{CI}_{95 \%}:[-0,39 ;-0,02]\right)$. Die durch die interindividuellen Unterschiede in der intraindividuellen Veränderung der Basic-Need-Satisfaction angenommenen indirekten Effekte des konstruktivistischen $\left(\beta_{\text {ind }}=0,01 ; S E_{\text {ind }}=0,03 ; p=\right.$ n. s.; $\mathrm{CI}_{95 \%}$ : $\left.[-0,04 ; 0,09]\right)$ und transmissiven $\left(\beta_{\text {ind }}=-0,01 ; S E_{\text {ind }}=0,03 ; p=\mathrm{n} . \mathrm{s} . ; \mathrm{CI}_{95 \%}\right.$ : $[-0,12 ; 0,04])$ Mentorings auf die interindividuellen Unterschiede in der intraindividuellen Veränderung der emotionalen Erschöpfung ist dagegen nicht signifikant.

\section{Diskussion}

Leitend für die vorliegende Studie war die Frage, inwiefern die jeweils implizierte anerkennende Beziehungsform des konstruktivistischen Mentorings oder hierarchische Beziehungsform des transmissiven Mentorings einen indirekten Effekt auf die positiven und negativen Beanspruchungsreaktionen angehender Lehrkräfte im Praxissemester hat, der durch die individuelle Basic-Need-Satisfaction vermittelt wird. Hinsichtlich der Basic-Need-Satisfaction konnte ein deutlicher Zuwachs beobachtet werden: Bei annähernd gleichverteilten Anteilen der Stichprobe hat sich die indivi- 
duelle Basic-Need-Satisfaction im Praxissemester nicht verändert oder ist gestiegen, nur sehr wenige Studierende erlebten hingegen eine Einschränkung ihrer BasicNeed-Satisfaction. Vergleichbar mit den Befunden von Römer et al. (2018) und Kücholl et al. (2019) verändert sich die emotionale Erschöpfung im Mittel zwar nicht, allerdings gibt es bedeutsame Unterschiede zwischen den Praxissemesterstudierenden. Die individuellen Differenzmaße zeigen Anteilswerte im Anstieg, Rückgang oder keiner Veränderung, die vergleichbar sind mit den Befunden von Schüle et al. (2017b) für das Praxissemester und von Dicke et al. (2015) für den Vorbereitungsdienst.

Entgegen den Befunden von Darge et al. (2018) erweist sich der berufliche Enthusiasmus als zeitstabil. Auch die Anteilswerte in den positiven und negativen Veränderungsgruppen liegen unter den von Darge et al. (2018) berichteten, deren Clusterung zu den beiden Messzeitpunkten aber auf der Skaleneinteilung in Gruppen mit niedriger, mittlerer und hoher Freude basiert, wodurch die Individualveränderung nur indirekt über den deskriptiven Übergang von der einen in die andere Gruppe operationalisiert wird. Dagegen wird mit den plausible values in der vorliegenden Studie ein direktes Differenzmaß verwendet, das die Signifikanz auf der Individualebene prüft. Darüber hinaus unterscheiden sich beide Studien in den verwendeten Skalen, weshalb die erfassten Konstrukte zur Operationalisierung der positiven Beanspruchungsreaktion nicht unmittelbar vergleichbar sind.

Die Ergebnisse zur Erklärung dieser Einzelbefunde anhand des angenommenen Wirkmechanismus legen eine teilweise Bestätigung der Forschungsfrage nahe: Wie mithilfe der Belastungs-Beanspruchungsmodelle im Lehrer/innenberuf (Böhm-Kasper 2004; Landwehr et al. 1983; Rudow 1994) sowie ihrer Ausdifferenzierung entlang der Selbstbestimmungstheorie von Ryan und Deci (2017) angenommen, können die anerkennende Beziehungsform des konstruktivistischen Mentorings und die hierarchische Beziehungsform des transmissiven Mentorings substanzielle Unterschiede in der Veränderung der Basic-Need-Satisfaction erklären (siehe zu ähnlichen Befunden: Hoffmann und Springer 2014; Simões und Alarcão 2014): Je hierarchischer die Praxissemesterstudierenden das Mentoring wahrnehmen, desto stärker geht auch ihre Basic-Need-Satisfaction über die untersuchte Zeitspanne des Praxissemesters zurück. Je anerkennender sie dagegen das Mentoring erleben, desto mehr nimmt ihre Basic-Need-Satisfaction zu. Diese interindividuellen Unterschiede in der intraindividuellen Veränderung der Basic-Need-Satisfaction klären wiederum bedeutsame Unterschiede in der intraindividuellen Veränderung des beruflichen Enthusiasmus auf: Je stärker die Basic-Need-Satisfaction der Studierenden zunimmt, desto größer fällt auch der Anstieg in ihrem beruflichen Enthusiasmus aus. Je eingeschränkter sie dagegen die Erfüllung ihrer Basic-Needs über die Zeit erleben, desto stärker nimmt auch ihr beruflicher Enthusiasmus ab. Im Hinblick auf die Unterschiede in der Veränderung der emotionalen Erschöpfung strebt jedoch der aufgeklärte Varianzanteil gegen Null. Dementsprechend stellt sich der angenommene indirekte Effekt der beiden Mentoringformen lediglich für den beruflichen Enthusiasmus ein. Damit stimmt dieses Ergebnis nicht mit den allgemeinen Befunden zur vermittelnden Bedeutung der Basic-Need-Satisfaction für den Zusammenhang zwischen der objektiven und subjektiven Belastung und einer daraus folgenden negativen Beanspruchungsreaktion überein (Aldrup et al. 2017; Fernet et al. 2013; Van den Broeck et al. 2008). 
Ein Grund für die gefundene Differentialität des Mediatoreffekts in Bezug auf die Vorhersage der Unterschiede in den Veränderungen der emotionalen Erschöpfung und des beruflichen Enthusiasmus könnte darin liegen, dass in dieser Studie nur eine der beiden möglichen kognitiven Bewertungen - die zielbezogene - direkt erfasst wurde. Möglicherweise könnte jedoch die bewältigungsbezogene Bewertung für die Erklärung der Veränderung der stressbezogenen Erschöpfung von höherer Bedeutung sein als in der vorliegenden Studie angenommen. Denn mit Radel et al. (2013) kann davon ausgegangen werden, dass dieser Bewertung eine moderierende Bedeutung im Hinblick auf den Einfluss der primären Bewertung auf die negativen Beanspruchungsreaktionen zukommt: Die hierarchische Beziehungsstruktur des transmissiven Mentorings könnte zwar die individuelle Befriedigung der Basic-Needs der angehenden Lehrkräfte einschränken, allerdings nur dann zu einer negativen Beanspruchungsreaktion führen, wenn angehende Lehrkräfte nicht über die Möglichkeiten verfügten, diese Reaktionen zu kompensieren. Die genauere Untersuchung einer moderierten Mediation durch den Einbezug von entsprechenden Konstrukten auf der bewältigungsbezogenen Bewertungsebene wäre deshalb eine lohnende Forschungsfrage.

Neben dieser konzeptionellen Einschränkung unterliegt die vorliegende Studie auch methodischen Limitationen wie den zum Teil nicht ganz zufriedenstellenden Modellfits der konfirmatorischen Faktorenanalysen. Außerdem umfasst die Untersuchungsstichprobe lediglich Lehramtsstudierende einer ausgewählten Universität. Darüber hinaus hat die Drop-Out-Analyse eine leichte geschlechtsbezogene Verzerrung dieser Stichprobe offengelegt, die in den Modellen bestmöglich berücksichtigt wurde. Generealisierte Aussagen lassen sich deshalb nicht treffen, sondern bedürfen weiterer Prüfungen. Ferner konnte das erlebte Mentoring der angehenden Lehrkräfte nur zum zweiten Messzeitpunkt erhoben werden. Damit haben die durchgeführten Mediatoranalysen im Rahmen des Parallel-Process-Modells nur einen querschnittlichen Charakter, sodass die Ergebnisse keine kausalen Schlüsse zulassen. Schließlich wurde das Mentoring allein aus der Perspektive der Mentees erhoben. Aus diesem Grund könnte in künftigen Studien die zusätzliche Erfassung der Mentor/ innenperspektive dabei helfen in Erfahrung zu bringen, welche Faktoren die Gestaltung und Wahrnehmung der jeweiligen Beziehungsform bedingen.

Trotz dieser methodischen Einschränkungen lässt sich eine praktische Implikation aus den Ergebnissen ableiten. Die gefundene Bedeutung des konstruktivistischen Mentorings spricht dafür, die anerkennende Beziehungsform nachdrücklich in den Fokus entsprechender Mentor/innenqualifikationen zu rücken und sich dort auf die Kenntnis und Erprobung von Strategien eines Need-Supportive Mentorings zu konzentrieren. Durch einen solchen Fokus könnten Aspekte lernwirksamen Mentorings zum Erwerb anwendbaren professionellen Wissens um Aspekte eines belastungsreduzierenden Mentorings erweitert und damit eine gezieltere Möglichkeit der proaktiven Unterstützung von Studierenden zur Abpufferung ihres Beanspruchungserlebens und Erhöhung ihres beruflichen Enthusiasmus beim späteren Berufseinstiegs geschaffen werden.

Funding Open Access funding enabled and organized by Projekt DEAL. 
Open Access Dieser Artikel wird unter der Creative Commons Namensnennung 4.0 International Lizenz veröffentlicht, welche die Nutzung, Vervielfältigung, Bearbeitung, Verbreitung und Wiedergabe in jeglichem Medium und Format erlaubt, sofern Sie den/die ursprünglichen Autor(en) und die Quelle ordnungsgemäß nennen, einen Link zur Creative Commons Lizenz beifügen und angeben, ob Änderungen vorgenommen wurden.

Die in diesem Artikel enthaltenen Bilder und sonstiges Drittmaterial unterliegen ebenfalls der genannten Creative Commons Lizenz, sofern sich aus der Abbildungslegende nichts anderes ergibt. Sofern das betreffende Material nicht unter der genannten Creative Commons Lizenz steht und die betreffende Handlung nicht nach gesetzlichen Vorschriften erlaubt ist, ist für die oben aufgeführten Weiterverwendungen des Materials die Einwilligung des jeweiligen Rechteinhabers einzuholen.

Weitere Details zur Lizenz entnehmen Sie bitte der Lizenzinformation auf http://creativecommons.org/ licenses/by/4.0/deed.de.

\section{Literatur}

Aldrup, K., Klusmann, U., \& Lüdtke, O. (2017). Does basic need satisfaction mediate the link between stress exposure and well-being? A diary study among beginning teachers. Learning and Instruction, 50, 21-30.

Asparouhov, T., \& Muthén, B. O. (2010). Plausible values for latent variables using Mplus. http://www. statmodel.com/download/Plausible.pdf. Zugegriffen: 4. Nov. 2019.

Böhm-Kasper, O. (2004). Schulische Belastung und Beanspruchung. Eine Untersuchung von Schülern und Lehrern am Gymnasium. Münster: Waxmann.

Van den Broeck, A., Vansteenkiste, M., de Witte, H., \& Lens, W. (2008). Explaining the relationships between job characteristics, burnout, and engagement: The role of basic psychological need satisfaction. Work \& Stress, 22(3), 277-294.

Darge, K., Valtin, R., Kramer, C., Ligtvoet, R., \& König, J. (2018). Die Freude an der Schulpraxis: Zur differenziellen Veränderung eines emotionalen Merkmals von Lehramtsstudierenden während des Praxissemesters. In J. König, M. Rothland \& N. Schaper (Hrsg.), Learning to Practice, Learning to Reflect? Ergebnisse aus der Längsschnittstudie LtP zur Nutzung und Wirkung des Praxissemesters in der Lehrerbildung (S. 241-264). Wiesbaden: Springer.

Dicke, T., Parker, P. D., Holzberger, D., Kunina-Habenicht, O., Kunter, M., \& Leutner, D. (2015). Beginning teachers' efficacy and emotional exhaustion. Latent changes, reciprocity, and the influence of professional knowledge. Contemporary Educational Psychology, 41, 62-72.

Ellis, N. J., Alonzo, D., \& Nguyen, H.T.M. (2020). Elements of a quality pre-service teacher mentor: a literature review. Teaching and Teacher Education, 92, 103072.

Evelein, F., Korthagen, F., \& Brekelmans, M. (2008). Fulfilment of the basic psychological needs of student teachers during their first teaching experiences. Teaching and Teacher Education, 24(5), 1137-1148.

Fernet, C., Austin, S., Trépanier, S.-G., \& Dussault, M. (2013). How do job characteristics contribute to burnout? Exploring the distinct mediating roles of perceived autonomy, competence, and relatedness. European Journal of Work and Organizational Psychology, 22(2), 123-137.

Haslam, C., Jetten, J., Cruwys, T., Dingle, G., \& Haslam, A. (2018). The new psychology of health. Unlocking the social cure. Florence: Routledge.

Hennissen, P., Crasborn, F., Brouwer, N., Korthagen, F., \& Bergen, T. (2008). Mapping mentor teachers' roles in mentoring dialogues. Educational Research Review, 3(2), 168-186.

Hoffmann, F., \& Springer, K. (2014). Wie Autonomie unterstützend agieren Mentorinnen und Mentoren bei ihrer Begleitung von Berufseinsteigerinnen und Berufseinsteigern? In G. Beer, I. Benischek, O. Dangl \& C. Plaimauer (Hrsg.), Mentoring im Berufseinstieg - eine mehrperspektivische Betrachtung. Erkenntnisse eines Entwicklungsprojekts (S. 57-95). Berlin: Lit.

Hu, L.-T., \& Bentler, P.M. (1999). Cutoff criteria for fit indexes in covariance structure analysis: conventional criteria versus new alternatives. Structural Equation Modeling: A Multidisciplinary Journal, 6(1), 1-55.

Keller, M.M., Goetz, T., Becker, E.S., Morger, V., \& Hensley, L. (2014). Feeling and showing. A new conceptualization of dispositional teacher enthusiasm and its relation to students' interest. Learning and Instruction, 33, 29-38.

Klassen, R. M., \& Durksen, T.L. (2014). Weekly self-efficacy and work stress during the teaching practicum: a mixed methods study. Learning and Instruction, 33, 158-169. 
Klusmann, U., Kunter, M., Voss, T., \& Baumert, J. (2012). Berufliche Beanspruchung angehender Lehrkräfte. Die Effekte von Persönlichkeit, pädagogischer Vorerfahrung und professioneller Kompetenz. Zeitschrift für pädagogische Psychologie, 26(4), 275-290.

König, J., Darge, K., \& Kramer, C. (2020). Kompetenzentwicklung im Praxissemester: Zur Bedeutung schulpraktischer Lerngelegenheiten auf den Erwerb von pädagogischen Wissen bei Lehramtsstudierenden. In I. Ulrich \& A. Gröschner (Hrsg.), Praxissemester im Lehramtsstudium in Deutschland: Wirkungen auf Studierende (S. 67-95). Wiesbaden: Springer.

Krawiec, V., Fischer, A., \& Hänze, M. (2020). Anforderungen und Erschöpfung während Schulpraktika im Lehramtsstudium. In I. Ulrich \& A. Gröschner (Hrsg.), Praxissemester im Lehramtsstudium in Deutschland: Wirkungen auf Studierende (S. 265-287). Wiesbaden: Springer.

Kücholl, D., Westphal, A., Lazarides, R., \& Gronostaj, A. (2019). Beanspruchungsfolgen Lehramtsstudierender im Praxissemester. Zeitschrift für Erziehungswissenschaft, 26(4), 519.

Landwehr, N., Fried, O., \& Hubler, P. (1983). Schulische Belastung. Problemstellung und theoretisches Konzept. Bildungsforschung und Bildungspraxis, 5(2), 125-146.

Lazarus, R.S. (1999). Stress and emotion. A new synthesis. New York: Springer.

Little, T. D., Rhemtulla, M., Gibson, K., \& Schoemann, A. M. (2013). Why the items versus parcels controversy needn't be one. Psychological Methods, 18(3), 285-300.

MacKinnon, D. P., Fairchild, A. J., \& Fritz, M. S. (2007). Mediation analysis. Annual Review of Psychology, 58, 593-614.

Maslach, C., \& Jackson, S. E. (1996). Maslach burnout inventory manual. Palo Alto: Consult. Psychological Press.

McArdle, J. J. (2009). Latent variable modeling of differences and changes with longitudinal data. Annual Review of Psychology, 60, 577-605.

Muthén, L. K., \& Muthén, B.O. (2014). Mplus 7.3 (Software). Los Angeles: Muthén \& Muthén.

Niedersächsisches Kultusministerium (2015p). Verordnung über Masterabschlüsse für Lehrämter in Niedersachsen (Nds. MasterVO-Lehr). http://www.nds-voris.de/jportal/?quelle=jlink\&query=MALehrV +ND\&psml=bsvorisprod.psml\&max=true\&aiz=true\#jlr-MALehrVND2015pP9. Zugegriffen: 20. Nov. 2020.

Radel, R., Pelletier, L., \& Sarrazin, P. (2013). Restoration processes after need thwarting: When autonomy depends on competence. Motivation and Emotion, 37(2), 234-244.

Reeve, J. (2009). Why teachers adopt a controlling motivating style toward students and how they can become more autonomy supportive. Educational Psychologist, 44(3), 159-175.

Richter, D., Kunter, M., Lüdtke, O., Klusmann, U., Anders, Y., \& Baumert, J. (2013). How different mentoring approaches affect beginning teachers' development in the first years of practice. Teaching and Teacher Education, 36, 166-177.

Römer, J., Rothland, M., \& Straub, S. (2018). Bedingungsfaktoren des Beanspruchungserlebens von Lehramtsstudierenden im Praxissemester. In J. König, M. Rothland \& N. Schaper (Hrsg.), Learning to Practice, Learning to Reflect? Ergebnisse aus der Längsschnittstudie LtP zur Nutzung und Wirkung des Praxissemesters in der Lehrerbildung (S. 265-286). Wiesbaden: Springer.

Rothland, M., \& Boecker, S. (2015). Viel hilft viel? Forschungsbefunde und -perspektiven zum Praxissemester in der Lehrerbildung. Lehrerbildung auf dem Prüfstand, 8(2), 112-134.

Rudow, B. (1994). Die Arbeit des Lehrers. Zur Psychologie der Lehrertätigkeit, Lehrerbelastung und Lehrergesundheit. Bern: Huber.

Ryan, R. M., \& Deci, E.L. (2017). Self-determination theory. Basic psychological needs in motivation, development, and wellness. New York: Guilford.

Scholl, D., Schüle, C., Feierabend, M., \& Küth, S. (2020). Motivationsregulation im Praxissemester - Zur Bedeutung assimilativer und akkommodativer Strategien für die intrinsische Studien- und Berufswahlmotivation. In K. Rheinländer \& D. Scholl (Hrsg.), Verlängerte Praxisphasen in der universitären Lehrerbildung: Spannungsfelder zwischen Theorie, Praxis und der Bestimmung von Professionalisierung (S. 151-165). Bad Heilbrunn: Klinkhardt.

Schüle, C., Besa, K.-S., Schriek, J., \& Arnold, K.-H. (2017a). Die Veränderung der Lehrerselbstwirksamkeitsüberzeugung in Schulpraktika. Zeitschrift für Bildungsforschung, 7(1), 23-40.

Schüle, C., Schriek, J., Kahlert, D., \& Arnold, K.-H. (2017b). Die Beanspruchungsregulation von Lehramtsstudierenden im Praxissemester. Lehrerbildung auf dem Prüfstand, 10(1), 91-103.

Simões, F., \& Alarcão, M. (2014). Promoting well-being in school-based mentoring through basic psychological needs support: does it really count? Journal of Happiness Studies, 15(2), 407-424.

Ulrich, I., Klingebiel, F., Bartels, A., Staab, R., Scherer, S., \& Gröschner, A. (2020). Wie wirkt das Praxissemester im Lehramtsstudium auf Studierende? Ein systematischer Review. In I. Ulrich \& A. Grösch- 
ner (Hrsg.), Praxissemester im Lehramtsstudium in Deutschland: Wirkungen auf Studierende (Bd. 9, S. 1-66). Wiesbaden: Springer.

Weyland, U. (2012). Expertise zu den Praxisphasen in der Lehrerbildung in den Bundesländern. Hamburg: LI. 